\title{
MODAL ANALYSIS OF A CRACKED BEAM
}

\author{
M. Chati, R. Rand and S. Mukherjee \\ Department of Theoretical and Applied Mechanics, Cornell University, Ithaca, \\ New York 14853, U.S.A.
}

\begin{abstract}
This paper addresses the problem of vibrations of a cracked beam. In general, the motion of such a beam can be very complex. This phenomenon can be attributed to the presence of the non-linearity due to the opening and closing of cracks. The focus of this paper is the modal analysis of a cantilever beam with a transverse edge crack. The non-linearity mentioned above has been modelled as a piecewise-linear system. In an attempt to define effective natural frequencies for this piecewise-linear system, the idea of a "bilinear frequency" is utilized. The bilinear frequency is obtained by computing the associated frequencies of each of the linear pieces of the piecewise-linear system. The finite element method is used to obtain the natural frequencies in each linear region.

In order better to understand the essential non-linear dynamics of the cracked beam, a piecewise-linear two-degree-of-freedom model is studied. Perturbation methods are used to obtain the non-linear normal modes of vibration and the associated period of the motion. Results of this piecewise-linear model problem are shown to justify the definition of the bilinear frequency as the effective natural frequency. It is therefore expected that calculating piecewise mode shapes and bilinear frequencies is useful for understanding the dynamics of the infinite degree of freedom cracked beam.
\end{abstract}

\section{INTRODUCTION}

Cracks are present in structures due to various reasons. The presence of a crack could not only cause a local variation in the stiffness, but it could affect the mechanical behavior of the entire structure to a considerable extent. Cracks present in vibrating/rotating components could lead to catastrophic failure. For these reasons, there is a need to understand the dynamics of cracked structures. The vibration characteristics of cracked structures can be useful for an on-line detection of cracks (non-destructive testing) without actually dismantling the structure. In particular, the natural frequencies and mode shapes of cracked beams can provide an insight into the extent of damage. As a representative model of a cracked structure, a cantilever beam with a transverse edge crack is studied in this paper.

In the past there have been considerable attempts to understand the dynamics of a cracked beam [1-9]. Christides and Barr [1] developed a one-dimensional cracked beam theory at the same level of approximation as the Bernoulli-Euler beam theory. Several assumptions on the displacement, velocity and stress fields are built into this model. The pair of symmetric cracks is always assumed to remain open as the beam is vibrating, so as to avoid the non-linear characteristics of an opening and closing crack. An approximate Galerkin solution to the one-dimensional cracked beam theory was obtained by Shen and Pierre [2]. The comparison functions used in the Galerkin procedure consisted of mode shapes of an uncracked beam. Shen and Chu [3] extended the cracked beam theory to account for the opening and closing of the crack-the so-called "breathing crack" model. 
A Galerkin procedure was used to obtain the bilinear equation for each vibration mode. The non-linear dynamic response of the bilinear equation to a forcing excitation was calculated through a numerical analysis.

Chu and Shen [4] obtained a closed form solution for a forced single-degree-of-freedom bilinear oscillator under low frequency excitation. They extended the procedure in order to study the dynamics of cracked beams with binlinear forcing functions. Yuen [5] proposed that the change in the stiffness of the cracked beam at the location of the crack can be modelled as a change in the modulus of elasticity of the cracked location. The finite element method was used to carry out the analysis. Shen and Taylor [6] developed an identification procedure for an on-line detection of the size and location of cracks. A mean square difference and a mini-max criterion were used to demonstrate the reliability of the identification procedure.

Ostachowicz and Krawczuk [7] replaced the crack section with a spring and then carried out modal analysis for each part of the beam using appropriate matching conditions at the location of the spring. The equivalent stiffness of the spring was calculated using the stress intensity factor at the crack location. Qian et al.[8] derived an element stiffness matrix of a beam with a crack, based on the integration of stress intensity factors. The finite element method was used to study the vibration response of the beam. Abraham and Brandon [9] modelled the opening and closing of a crack using a substructuring approach. Lagrange multipliers and time varying connection matrices were used to represent the interaction forces between the two segments of the cantilever beam separated by the crack. The effect of dry friction when the crack is closed has been accounted for in this model.

A cracked vibrating beam has an inherent non-linearity present in it due to the opening and closing of the crack. Not all of the papers mentioned above model this non-linearity. For example, some authors assume that a crack always remains open. In this paper, we explicitly account for this non-linear behaviour of the beam by not allowing interpenetration of the crack faces. Some of the distinctive features of this paper are: (i) modelling of the opening and closing of the crack as a multi-degree-of-freedom piecewise-linear system; (ii) definition of an effective natural frequency, called the "bilinear frequency"; and (iii) a systematic study of a two-degree-of-freedom piecewise-linear model to understand the essential non-linear dynamics.

The paper is organized as follows. In section 2, we describe the modal analysis procedure for obtaining the effective natural frequencies for the cracked beam. In section 3 , we give the numerical results of the finite element method used to carry out the analysis. In section 4 , we begin studying the bilinear two-degree-of-freedom oscillator, and in section 5 , we present a brief introduction to non-linear normal modes. In section 6 , a perturbation method is used to obtain expressions for the non-linear normal modes of the bilinear system, and in section 7 our results are compared with the results of numerical integration. Our conclusions are presented in section 8 .

\section{MODAL ANALYSIS}

Consider a uniform cantilever beam, of length $L$, having a transverse crack at location $b$ of depth $a$ (Figure 1). One can qualitatively see that the global stiffness of the cracked beam would depend on whether the crack is open or closed. In the past (see references $[3,4])$, the cracked beam has been modelled as a bilinear single-degree-of-freedom system (Figure 2). In what follows, we shall briefly describe the procedure for obtaining the "bilinear frequency" of this piecewise-linear single-degree-of-freedom system. 


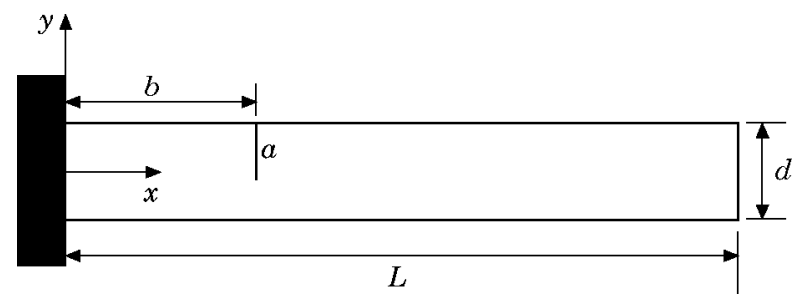

Figure 1. A cantilever beam with a transverse edge crack, where $a$ is the length of the crack, $b$ is the location of the crack, $L$ is the length of the beam and $d$ is the depth of the beam.

\subsection{ONE-DEGREE-OF-FREEDOM SYSTEM}

The system shown in Figure 2 consists of a mass $m$ which is attached to a linear spring $k_{1}$ and which makes contact with the linear spring $k_{2}$ only when $x<0$. The equations of motion for this system are

$$
\begin{array}{cc}
m \ddot{x}+k_{1} x=0 & \text { for } x>0, \\
m \ddot{x}+\left(k_{1}+k_{2}\right) x=0 & \text { for } x<0,
\end{array}
$$

where the dots indicate differentiation with respect to time. The free vibration problem can be easily solved analytically (see, for example, reference [4]). However, the solution is presented here for the sake of completeness. Before we solve the free vibration problem, let us solve for the two natural frequencies in the two linear regions, $x>0$ and $x<0$. The two frequencies are

$$
\omega_{1}=\sqrt{\frac{k_{1}}{m}} \quad \text { for } \quad x>0, \quad \omega_{2}=\sqrt{\frac{k_{1}+k_{2}}{m}} \quad \text { for } x<0 .
$$

To solve equations (1) and (2), let the initial conditions be $x(0)=x_{0}$ and $\dot{x}(0)=0$. Without loss of generality, let us assume that $x_{0}>0$. The solution to equation (1) is

$$
x(t)=x_{0} \cos \omega_{1} t \quad \text { for } t<t_{1} \quad(\text { here } x>0) .
$$

At time $t_{1}=\pi / 2 \omega_{1}, x\left(t_{1}\right)=0$ and the mass $m$ enters the region $x<0$ with the new initial conditions $x\left(t_{1}\right)=0$ and $\dot{x}\left(t_{1}\right)=-x_{0} \omega_{1}$. The solution to the equation (2) is given as

$$
\left.x(t)=-\frac{x_{0} \omega_{1}}{\omega_{2}} \sin \omega_{2}\left(t-t_{1}\right) \quad \text { for } t_{1} \leqslant t \leqslant t_{2} \quad \text { (here } x<0\right) .
$$

At time $t_{2}=t_{1}+\pi / \omega_{2}, x\left(t_{2}\right)=0$ and the mass $m$ enters the region $x>0$ with the initial conditions $x\left(t_{2}\right)=0$ and $\dot{x}\left(t_{2}\right)=x_{0} \omega_{1}$. Note that the velocity with which the mass $m$ enters the region $x<0$ is exactly the same in magnitude as the velocity with which it left the region $x<0$. The solution to equation (1) now is

$$
x(t)=x_{0} \sin \omega_{1}\left(t-t_{2}\right) \quad \text { for } t_{2} \leqslant t \leqslant t_{3} \quad(\text { here } x>0) .
$$

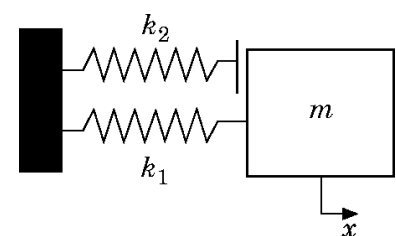

Figure 2. The one-degree-of-freedom system. 
At the time $t_{3}=t_{2}+\pi / 2 \omega_{1}, x\left(t_{3}\right)=x_{0}$ and $\dot{x}\left(t_{3}\right)=0$; i.e., the mass $m$, comes back exactly to the same point in the state space $(x, \dot{x})$ from where the motion started at $t=0$. Thus, the motion is periodic and the effective period $\left(T_{0}\right)$ of this piecewise-linear system is

$$
T_{0}=t_{3}=\frac{\pi}{\omega_{1}}+\frac{\pi}{\omega_{2}}=\frac{2 \pi}{\omega_{0}},
$$

where the effective natural frequency $\omega_{0}$ of this piecewise-linear single-degree-of-freedom system is defined as

$$
\omega_{0}=\frac{2 \omega_{1} \omega_{2}}{\omega_{1}+\omega_{2}}
$$

The effective natural frequency $\omega_{0}$ is called the "bilinear frequency". The central idea of this paper is to extend the definition of bilinear frequency from the single-degree-of-freedom bilinear system to the infinite-degree-of-freedom cracked beam problem. Just as the bilinear system (Figure 2) has two linear regions $(x>0$ and $x<0$ ), the cracked beam problem can be modelled as having two configurations: crack open and crack closed. In each of the individual linear regions, one can define a set of natural frequencies by solving an eigenvalue problem. The two sets of natural frequencies can then be combined, using equation (8), to obtain the effective natural frequencies for the cracked beam. In what follows we shall describe the procedure for calculating the frequencies for the two configurations of the cracked beam.

\subsection{CRACKED BEAM}

The eigenvalues of the cracked beam in the two configurations (crack open and crack closed) can be extracted using a numerical method. Among the various numerical methods present, the most popular are the finite difference method (FDM), the finite element method (FEM) and the boundary element method (BEM). The eigenvalue problem of the cracked beam (Figure 1) is solved using the FEM in this paper.

The basic idea of the finite element method (see reference [10]) is to discretize a body into an assemblage of discrete finite elements which are interconnected at the nodal points on element boundaries. The displacement field is approximated over each finite element, in terms of the nodal displacements. The equations of motion can then be formulated using the displacement-based formulation in conjunction with the principle of virtual displacements. These equations of motion can be written as

$$
\mathbf{M U ̈}+\mathbf{K U}=\mathbf{0},
$$

where overdots indicate differentiation with respect to time, $\mathbf{M}$ and $\mathbf{K}$ are the mass and stiffness matrix respectively, and $\mathbf{U}\left(=\left[u_{1} v_{1} u_{2} v_{2} \ldots u_{n} v_{n}\right]^{\mathrm{T}}\right)$ is the column vector of nodal displacements. Each node $i$ has two degrees of freedom, $u_{i}$ and $v_{i}$, in the horizontal $(x)$ and vertical $(y)$ directions, respectively.

To explain the idea of the two configurations used to calculate the natural frequencies, consider the two crack faces shown in Figure 3. The numbers on the left and right faces of the crack are the node numbers of the interacting nodes. For the first configuration, assume that the crack faces remain open which basically implies that the degrees of freedom associated with the nodes on the crack surfaces are independent. This configuration will be referred to as the "unconstrained" configuration. For the second configuration, assume that the crack is closed. Now, to prevent a physically unrealistic situation with interpenetration of crack faces, the horizontal degrees of freedom $\left(u_{i}\right)$ of 


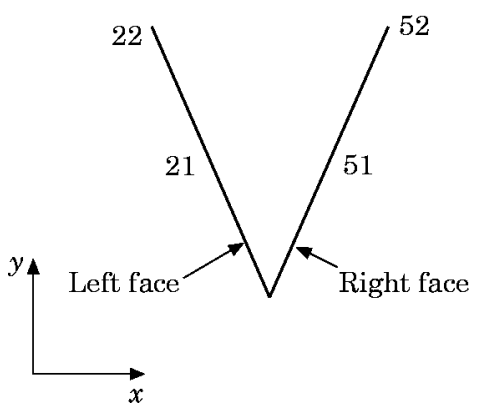

Figure 3. Crack faces.

the nodes on the left and right faces of the crack, which are initially at the same geometric location, need to be restricted. This condition can be stated as

$$
u_{l}^{\text {leffface }}=u_{r}^{\text {rightface }},
$$

where node numbers $l$ and $r$ are the corresponding interacting nodes on the left and right face of the crack. This implies that the imposed virtual displacements must also obey the constraint (10). This configuration will be referred to as the "constrained" configuration. The effect of this constraint can be taken into account by an appropriate addition of the corresponding rows and columns of the mass and stiffness matrices. Note that there is no constraint in the vertical direction $\left(v_{i}\right)$ on the crack faces. Therefore, there could be frictionless sliding of the crack faces, and the closed crack configuration need not correspond to an uncracked beam, as assumed in some previous research (see, for example, reference [3]).

Let us assume a time harmonic solution for the nodal displacements:

$$
\mathbf{U}=\mathbf{A} \sin \omega t,
$$

where $\mathbf{A}$ is the amplitude of the nodal displacements. Substituting equation (11) into equation (9) leads to

$$
\left[-\omega^{2} \mathbf{M}+\mathbf{K}\right]\{\mathbf{A}\}=\mathbf{0} .
$$

For non-trivial solutions,

$$
\operatorname{det}\left(\mathbf{K}-\omega^{2} \mathbf{M}\right)=0,
$$

where "det" denotes the determinant and $\omega$ is the natural frequency.

Using equation (13) for the two configurations of the cracked beam, two sets of natural frequencies can be obtained. Let us denote the mass and stiffness matrices corresponding to the unconstrained configuration with the subscript 1 and that of the constrained configuration with subscript 2. For the unconstrained configuration, the eigenvalue problem takes the form

$$
\operatorname{det}\left(\mathbf{K}_{\mathbf{1}}-\omega_{1 i}^{2} \mathbf{M}_{\mathbf{1}}\right)=0,
$$

where $\omega_{1 i}$ is the set of natural frequencies corresponding to the unconstrained configuration arranged in order of increasing magnitude. Similarly, for the constrained configuration,

$$
\operatorname{det}\left(\mathbf{K}_{2}-\omega_{2 i}^{2} \mathbf{M}_{2}\right)=0,
$$

where $\omega_{2 i}$ is the set of natural frequencies corresponding to the constrained configuration arranged in the order of increasing magnitude. 
In this work, we define an effective natural frequency, called the bilinear frequency, which permits us to obtain an approximation for the frequency associated with the $i$ th mode of a piecewise-linear vibrating system without computing the actual mode shape. The bilinear frequency is obtained by computing the associated frequencies of each of the linear pieces of the piecewise-linear system. The important question that we want to address is the relationship between the $i$ th mode shape and natural frequency to those obtained from a "simplified" finite element analysis, where one computes the mode shapes and frequencies of the individual linear segments of the piecewise-linear system. We call the latter the "bilinear mode shapes". It is expected that if the crack is sufficiently shallow, the two results, namely the actual and bilinear $i$ th mode shapes, will be close to each other. In such a case, we would also expect that the overall frequency of the $i$ th mode will be well approximated by the bilinear frequency as we define it. According to this line of reasoning, calculation of these bilinear mode shapes and frequencies for a vibrating cracked beam appears meaningful. This is done later in this paper. Also, the actual and bilinear mode shapes are computed and compared for a simplified two-degree-of-freedom model of the cracked beam. It is seen that the bilinear mode shapes are comparable to the actual ones. Details are given later in the paper.

The two sets of frequencies $\left(\omega_{1 i}\right.$ and $\left.\omega_{2 i}\right)$ for the two configurations of the cracked beam can be combined, using equation (8), to define effective natural frequencies $\left(\Omega_{i}\right)$ of the cracked beam as

$$
\Omega_{i}=\frac{2 \omega_{1 i} \omega_{2 i}}{\omega_{1 i}+\omega_{2 i}}
$$

\section{NUMERICAL RESULTS AND DISCUSSION}

Due to the presence of the crack, the beam cannot be modelled using beam elements; but, rather, we need to use plate/shell elements. The cracked cantilever beam has been modelled as a problem in plane stress using eight-noded quadrilateral plate elements. The idea of using beam elements and plate elements in conjunction with transition elements was considered, but since the amount of computational saving was limited the idea was not pursued further. The commercially available finite element package ABAQUS was used to obtain the numerical results. The material and geometric parameters used to carry out the analysis are: $E=210 \mathrm{GPa}, \rho=7850 \mathrm{~kg} / \mathrm{m}^{3}, v=0 \cdot 3, L=10 \mathrm{~m}$ and $A=1 \mathrm{~m}^{2}$. The location $b$ and the length $a$ of the crack have been varied to study their effect on the natural frequencies of the cracked beam.

First, a convergence study is presented in Tables 1 and 2 for a crack location of $b=5 \mathrm{~m}$ and $a=0.5 \mathrm{~m}$. Table 1 contains the frequencies for the unconstrained configuration and Table 2 contains the frequencies for the constrained configuration. It can be seen that the effect of increasing the number of elements from 192 to 704 does not have a significant effect on the natural frequencies for the first few modes. All further calculations have been carried out using 704 elements. The undeformed mesh consisting of 704 elements, with $b=5 \mathrm{~m}$ and $a=0.5 \mathrm{~m}$, is shown in Figure 4.

Due to the presence of the crack, there is a $1 / \sqrt{r}$ singularity in the stress field at the crack tip. Usually, quarter-point elements are used to model the singular behavior in the stress field. However, in this particular analysis, global changes in the dynamics of the beam are desired, rather than the local variation in the stress field due to the presence of the crack. A comparison in natural frequencies when the singular elements are used and when they are not used is presented in Table 3. The results presented in this table are the natural frequencies for the unconstrained configuration of the beam with $b=2 \mathrm{~m}$ and 
TABLE 1

A convergence study for natural frequencies ( $\mathrm{rad} / \mathrm{s})$ in the unconstrained configuration

\begin{tabular}{lrrrrr}
\hline \multicolumn{5}{c}{ Number of elements } \\
\cline { 2 - 6 } & \multicolumn{1}{c}{4} & \multicolumn{1}{c}{20} & \multicolumn{1}{c}{40} & \multicolumn{1}{c}{192} & \multicolumn{1}{c}{704} \\
\hline First transverse mode & $53 \cdot 40$ & $49 \cdot 83$ & $49 \cdot 63$ & $48 \cdot 33$ & $48 \cdot 24$ \\
Second transverse mode & $385 \cdot 17$ & $266 \cdot 51$ & $263 \cdot 79$ & $247 \cdot 24$ & $246 \cdot 40$ \\
First longitudinal mode & $789 \cdot 27$ & $768 \cdot 75$ & $765 \cdot 41$ & $746 \cdot 61$ & $745 \cdot 88$ \\
Third transverse mode & $1165 \cdot 65$ & $827 \cdot 83$ & $826 \cdot 25$ & $825 \cdot 07$ & $820 \cdot 98$ \\
Fourth transverse mode & $2331 \cdot 80$ & $1369 \cdot 1$ & $1361 \cdot 9$ & $1334 \cdot 60$ & $1324 \cdot 40$ \\
\hline
\end{tabular}

TABLE 2

A convergence study for natural frequencies $(\mathrm{rad} / \mathrm{s})$ in the constrained configuration

\begin{tabular}{lcrrrr}
\hline \multicolumn{5}{c}{ Number of elements } \\
\cline { 2 - 6 } & \multicolumn{1}{c}{4} & \multicolumn{1}{c}{20} & \multicolumn{1}{c}{40} & \multicolumn{1}{c}{192} & 704 \\
\hline First transverse mode & $54 \cdot 31$ & $52 \cdot 09$ & $52 \cdot 18$ & $52 \cdot 12$ & $52 \cdot 03$ \\
Second transverse mode & $424 \cdot 21$ & $309 \cdot 76$ & $312 \cdot 34$ & $311 \cdot 08$ & $310 \cdot 13$ \\
First longitudinal mode & $815 \cdot 97$ & $806 \cdot 13$ & $811 \cdot 84$ & $809 \cdot 82$ & $809 \cdot 41$ \\
Third transverse mode & $1177 \cdot 6$ & $827 \cdot 94$ & $826 \cdot 38$ & $825 \cdot 18$ & $821 \cdot 07$ \\
Fourth transverse mode & $2492 \cdot 70$ & $1496 \cdot 00$ & $1503 \cdot 30$ & $1501 \cdot 60$ & $1487 \cdot 10$ \\
\hline
\end{tabular}

TABLE 3

A comparison of natural frequencies ( $\mathrm{rad} / \mathrm{s})$ for singular versus non-singular elements

\begin{tabular}{lrc}
\hline & Singular & Non-singular \\
\hline First transverse mode & $39 \cdot 62$ & $39 \cdot 47$ \\
Second transverse mode & $311 \cdot 72$ & 311.68 \\
First longitudinal mode & $688 \cdot 57$ & 666.96 \\
Third transverse mode & 818.76 & 818.67 \\
Fourth transverse mode & $1381 \cdot 10$ & $1380 \cdot 50$ \\
\hline
\end{tabular}

$a=0.5 \mathrm{~m}$. The frequencies are quite close. Therefore, we decided not to use singular quarter-point elements in further calculations.

The mode shapes of the beam, with $b=5 \mathrm{~m}$ and $a=0.5 \mathrm{~m}$, in the two configurations are shown in Figures 5 and 6. In Figures 5(a), (b) and (c) are shown the first, second and third modes, respectively, for the unconstrained configuration of the beam, while in Figures 6(a), (b) and (c) are shown the first, second and third modes for the constrained configuration of the beam. These mode shapes will be utilized to explain the trends in the effective bilinear frequencies with the variation of parameters such as crack location $b$ and crack length $a$.

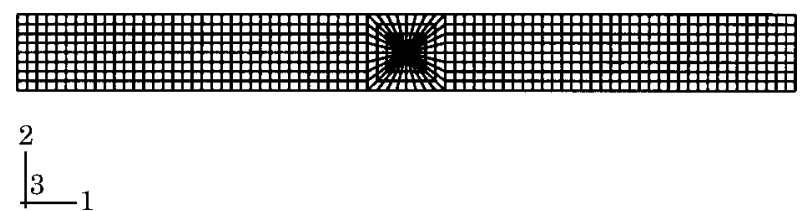

Figure 4. The undeformed mesh. 

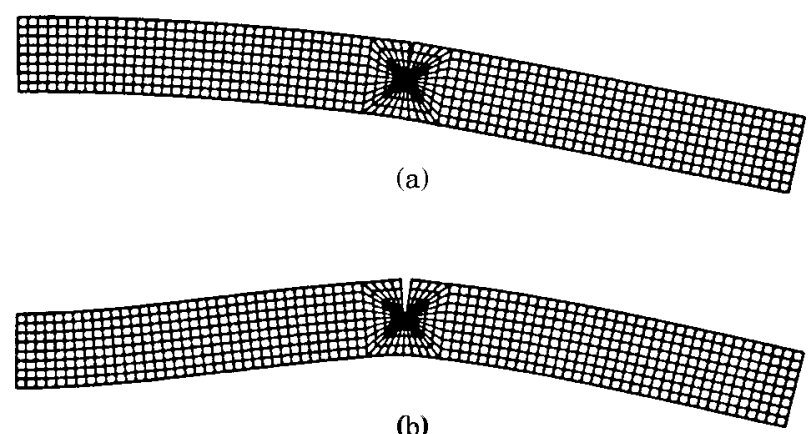

(b)

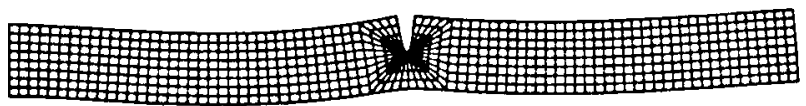

(c)

Figure 5. Unconstrained mode shapes for the (a) first, (b) second and (c) third mode shapes.

In Figure 7 is shown the variation in the normalized first bilinear effective frequency with the normalized crack depth $a / d$. The variation shown in Figure 7 can be easily understood if we look at the corresponding mode shapes associated with the first mode (see Figures 5(a) and 6(a)). Since in the first mode of vibration the major contribution to the stiffness comes from the clamped end of the beam, a crack present at $b / L=0 \cdot 2$ results in a substantial decrease in the bilinear frequencies, as compared to $b / L=0.5$ or $0 \cdot 7$. The variation in the second and third effective bilinear frequencies with crack depth is shown in Figures 8 and 9.

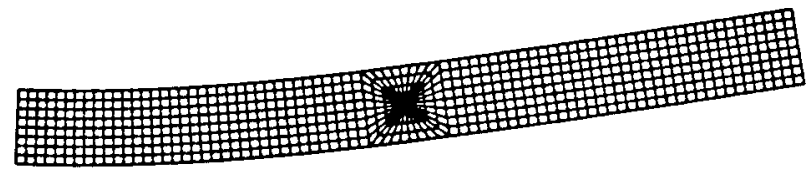

(a)

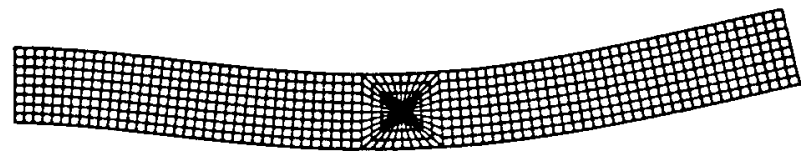

(b)

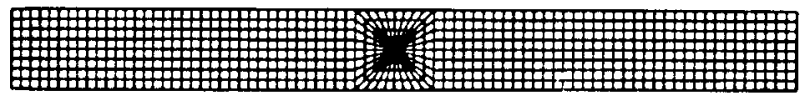

(c)

Figure 6. Constrained mode shapes for the (a) first, (b) second and (c) third mode shapes. 


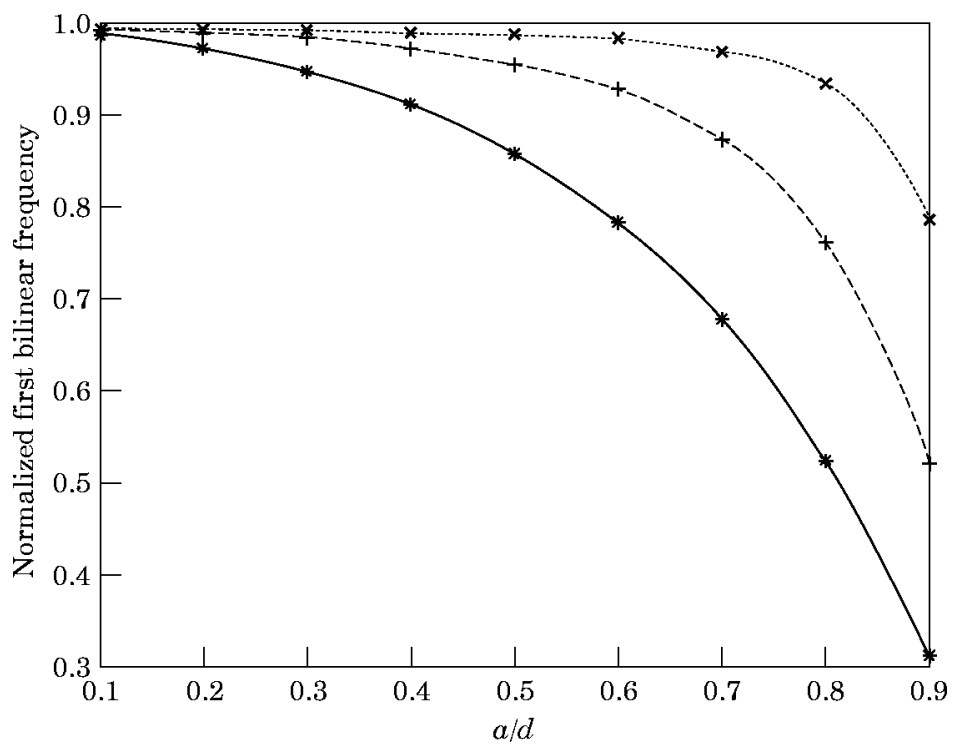

Figure 7. The variation of the first bilinear frequency with crack depth, where $b$ is the location of the crack and $L$ is the length of the beam. ${ }^{*}, b / L=0 \cdot 2 ;+, 0 \cdot 5 ; \times, 0 \cdot 7$.

In order to justify the intuitive assertion that the bilinear frequency is a good approximation for the effective natural frequency of the multi-degree-of-freedom piecewise-linear system we next investigate a two-degree-of-freedom piecewise-linear system using perturbation methods. It is shown that the bilinear frequency a good approximation to the frequencies of the (non-linear) normal modes in this case.

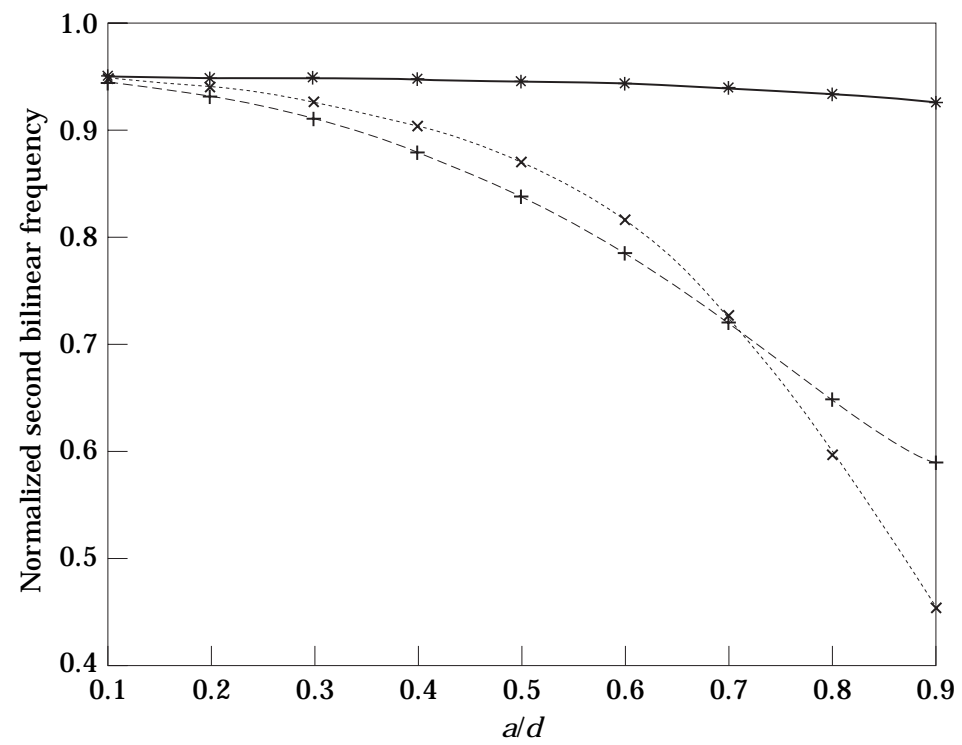

Figure 8. The variation of the second bilinear frequency with crack depth, where $b$ is the location of the crack and $L$ is the length of the beam. ${ }^{*}, b / L=0 \cdot 2 ;+, 0 \cdot 5 ; \times, 0 \cdot 7$. 


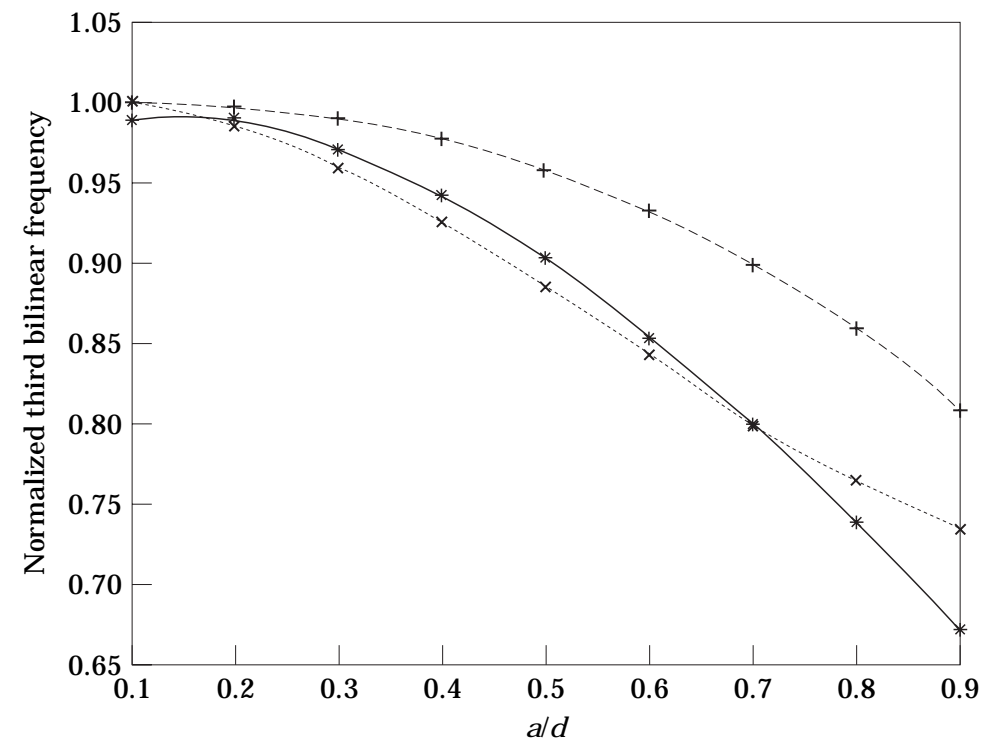

Figure 9. The variation of the third bilinear frequency with crack depth, where $b$ is the location of the crack and $L$ is the length of the beam. ${ }^{*}, b / L=0 \cdot 2 ;+, 0 \cdot 5 ; \times, 0 \cdot 7$.

\section{TWO-DEGREE-OF-FREEDOM SYSTEM}

In order better to understand the essential nonlinear dynamics of the cracked beam, we study in this section a simplified piecewise-linear two-degree-of-freedom (2-DOF) system. The system is displayed in Figure 10 and consists of two masses constrained to a straight line and restrained by three springs. Note that spring $k_{2}$ applies no force to mass $m_{1}$ unless co-ordinate $x_{1}<0$. This feature represents, in this simplified model, the essential character of the cracked beam. The equations of motion for this system are

$$
m_{1} \ddot{x}_{1}+\left(k_{1}+k_{3}+k_{2} \mathrm{H}\left(x_{1}\right)\right) x_{1}-k_{3} x_{2}=0, \quad m_{2} \ddot{x}_{2}-k_{3} x_{1}+k_{3} x_{2}=0,
$$

where $x_{1}$ and $x_{2}$ are the displacements of the masses $m_{1}$ and $m_{2}$ respectively, dots indicate differentiation with respect to time, and

$$
\mathrm{H}\left(x_{1}\right)= \begin{cases}1, & x_{1}<0 \\ 0, & x_{1}>0\end{cases}
$$

As the mass $m_{1}$ goes through $x_{1}=0$, this system switches between each of the two linear problems, one for $x_{1}>0$ and another for $x_{1}<0$. We now obtain the corresponding two

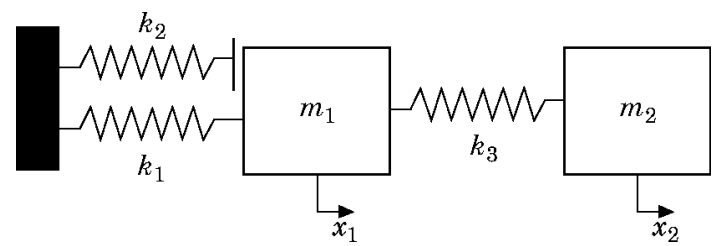

Figure 10. The two-degree-of-freedom model. 
sets of eigenvalues and eigenvectors, which will be used in what follows. For $x_{1}>0$, the characteristic equation is

$$
\lambda^{2}-\left[\frac{k_{1}+k_{3}}{m_{1}}+\frac{k_{3}}{m_{2}}\right] \lambda+\frac{k_{1} k_{3}}{m_{1} m_{2}}=0,
$$

where $\lambda=\omega^{2}$. Equation (19) can be solved for the two natural frequencies $\left(\omega_{11}\right.$ and $\left.\omega_{12}\right)$ and then the corresponding eigenvectors can be obtained. These frequencies would actually be the fundamental frequencies of the system in the absence of the spring $k_{2}$.

Carrying out a similar analysis for the case when $x_{1}<0$, the characteristic equation takes the form

$$
\lambda^{2}-\left[\frac{k_{1}+k_{2}+k_{3}}{m_{1}}+\frac{k_{3}}{m_{2}}\right] \lambda+\frac{\left(k_{1}+k_{2}\right) k_{3}}{m_{1} m_{2}}=0
$$

which can be solved for the two natural frequncies $\left(\omega_{21}\right.$ and $\left.\omega_{22}\right)$ and the corresponding mode shapes.

The eigenfrequencies in the two regions can be combined in pairs to form two effective bilinear frequencies for this non-linear system. These are given by the same formula used to define the effective bilinear frequency for a one-DOF bilinear system. The bilinear frequencies are defined as

$$
\Omega_{1}=\frac{2 \omega_{11} \omega_{21}}{\omega_{11}+\omega_{21}}, \quad \Omega_{2}=\frac{2 \omega_{12} \omega_{22}}{\omega_{12}+\omega_{22}} .
$$

In the next two sections we will use perturbation methods and numerical integration to show that the frequencies for the non-linear normal modes associated with the system shown in Figure 10 are well approximated by the bilinear frequencies (21) and (22).

\section{NON-LINEAR NORMAL MODES}

Non-linear normal modes are a class of special solutions of $n$-DOF conservative systems. These could be considered as a non-linear analog of the classical normal modes in a linear system. They were shown by Rosenberg [11] to be important because resonance in the forced system typically occurs in the neighborhood of the non-linear normal modes of the free system. Over the years since Rosenberg's original work, there have been many studies of non-linear normal modes. An outstanding recent book by Vakakis et al. [12] summarizes much of the research in this area to date. We mention here a few works of non-linear normal modes, but the reader is referred to reference [12] for a complete bibliography.

Rand [13] obtained explicit approximate expressions for normal modal curves in non-linear two-degree-of-freedom systems by applying a perturbation method to the modal equation. Rand et al. [14] studied the stability and bifurcation of non-linear normal modes in a class of two-degree-of-freedom systems. King and Vakakis [15] developed an energy-based formulation for computing non-linear normal modes in undamped continuous systems. A partial differential equation is constructed using conservation of energy, which is solved asymptotically using a perturbation methodology. Nayfeh et al. [16] used two approaches to obtain the non-linear normal modes of a cantilever beam. The same non-linear normal mode is obtained by the application of the method of multiple scales to the discretized problem and the original boundary value problem. Shaw and Pierre [17] extended the concept of non-linear normal modes to a multi-degree-of-freedom 
system with damping. They used the theory of invariant manifolds in conjunction with the center manifold reduction technique.

In the problem at hand, the concept of non-linear normal modes must be applied to a piecewise-linear system. This topic has been studied by Chen and Shaw [19], who presented a general discussion of non-linear normal modes in piecewise-linear systems and an example of a 2-DOF system which is similar (but not identical) to the 2-DOF system studied in our paper. They obtained the non-linear normal modes using an invariant manifold approach in the four-dimensional space. In contrast to their approach, we present an analysis of a 2-DOF piecewise-linear system based on a modal equation in the two-dimensional configuration space.

We will define non-linear normal modes as vibrations in unison; i.e., synchronous periodic motions in which all generalized coordinates vibrate equiperiodically, achieving instantaneous zero velocity at the same instant of time. (This is a slight generalization of Rosenberg's definition, suitable for the piecewise-linear system at hand.)

In order to obtain the non-linear modes for the system in Figure 10, we will use Rosenberg's modal equation, derived as follows. Consider a conservative system for which the potential energy $V$ is of the form

$$
V=\frac{1}{2}\left(a x^{2}+2 b x y+c y^{2}\right),
$$

where $x$ and $y$ are the generalized co-ordinates. The constants $a, b$ and $c$ are such that $V$ is positive definite, and kinetic energy $T$ is of the form

$$
T=\frac{1}{2}\left(\dot{x}^{2}+\dot{y}^{2}\right) .
$$

Then

$$
\ddot{x}=-V_{x}, \quad \ddot{y}=-V_{y}, \quad T+V=h,
$$

where $h$ is a constant equal to the total energy of the system. In what follows, we will eliminate time $t$ by considering $y$ as a function of $x$ only. Using equations (25-27) and

$$
\dot{y}=y^{\prime} \dot{x}, \quad \ddot{y}=y^{\prime \prime} \dot{x}^{2}+y^{\prime} \ddot{x},
$$

we obtain the governing equation for the normal modes of the system,

$$
2(h-V) y^{\prime \prime}+\left(1+y^{\prime 2}\right)\left(V_{y}-y^{\prime} V_{x}\right)=0,
$$

where the primes denote differentiation with respect to $x$. We will define a non-linear normal mode to a be a solution to equation (30) which is single valued and which has two rest points; i.e., two points at which $\dot{x}$ and $\dot{y}$ simultaneously vanish giving $T=0$, or, from equation (27), $V=h$. From equation (30), at such points where $V=h$, we find that

$$
V_{y}-y^{\prime} V_{x}=0 \quad \text { at } V=h .
$$

Geometrically, this implies that a non-linear normal mode intersects the bounding curve $V=h$ orthogonally at two points, as shown in Figure 11. In what follows we shall use a perturbation approach to obtain the nonlinear normal modes of the bilinear two degree of freedom system (Figure 10).

\section{PERTURBATION METHOD}

We begin by expressing the equations (17) and (18) in normal co-ordinates $x$ and $y$ for the $x_{1}>0$ system. The modal matrix consisting of orthonormal eigenvectors obtained for 


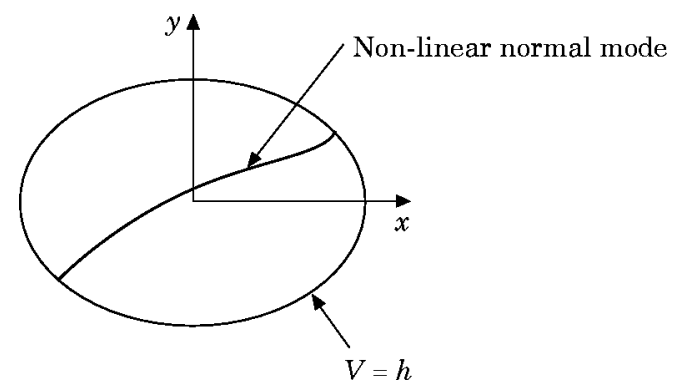

Figure 11. The non-linear normal mode.

the region $x_{1}>0$ is used to achieve the transfer to normal co-ordinates. This transformation can be written in the abbreviated form as

$$
\left\{\begin{array}{l}
x_{1} \\
x_{2}
\end{array}\right\}=\left[\begin{array}{ll}
a_{1} & a_{2} \\
a_{3} & a_{4}
\end{array}\right]\left\{\begin{array}{l}
x \\
y
\end{array}\right\}
$$

The coefficients $a_{i}$ are the components of the eigenvectors and are not listed here for brevity. Note that we will be using the same modal matrix to affect the transformation to the normal co-ordinates for both $x_{1}>0$ and $x_{1}<0$. The equations of motion for the region $\left(x_{1}>0\right)$ in the normal co-ordinates then take the form

$$
\ddot{x}=-\omega_{11}^{2} x, \quad \ddot{y}=-\omega_{12}^{2} y .
$$

The potential energy is of the form

$$
V=\frac{1}{2}\left(\omega_{11}^{2} x^{2}+\omega_{12}^{2} y^{2}\right)
$$

and the kinetic energy has the same form as equation (24).

The equations of motion for the region $x_{1}<0$ in the normal co-ordinates take the form

$$
\ddot{x}=-\left(\omega_{11}^{2}+a_{1}^{2} k_{2}\right) x-a_{1} a_{2} k_{2} y, \quad \ddot{y}=-a_{1} a_{2} k_{2} x-\left(\omega_{12}^{2}+a_{2}^{2} k_{2}\right) y .
$$

The potential energy is of the form

$$
V=\frac{1}{2}\left(\omega_{11}^{2} x^{2}+\omega_{12}^{2} y^{2}\right)+\frac{k_{2}}{2}\left(a_{1}^{2} x^{2}+2 a_{1} a_{2} x y+a_{2}^{2} y^{2}\right)
$$

and the kinetic energy has the same form as equation (24). Note that the presence of an $x y$ term in the equation (38) is due to the fact that while co-ordinates $x$ and $y$ are normal co-ordinates for $x_{1}>0$, they are not normal co-ordinates for $x_{1}<0$.

A perturbation method will be used to solve equation (30) approximately. We will assume that the spring $k_{2}$ is small:

$$
k_{2}=k \varepsilon, \quad \varepsilon \ll 1,
$$

where $\epsilon$ is a small parameter. The non-linear normal mode that we desire to solve for can be taken to be a perturbation to the linear mode $(y=0)$. To solve for a non-linear normal mode $y(x)$, we assume

$$
y(x)=\left\{\begin{array}{ll}
\varepsilon f_{1}(x), & x_{1}>0, \\
\varepsilon f_{2}(x), & x_{1}<0
\end{array} .\right.
$$


Substituting equation (40) into the modal equation (30), i.e., with $V$ as in equation (35), we obtain, neglecting terms of order $\varepsilon$, the differential equation governing the non-linear normal mode for the region $x_{1}>0$ :

$$
\left(2 h-\omega_{11}^{2} x^{2}\right) f_{1}^{\prime \prime}-\omega_{11}^{2} x f_{1}^{\prime}+\omega_{12}^{2} f_{1}=0+O(\varepsilon) .
$$

Similarly, for the region $x_{1}<0$ we obtain

$$
\left(2 h-\omega_{11}^{2} x^{2}\right) f_{2}^{\prime \prime}-\omega_{11}^{2} x f_{2}^{\prime}+\omega_{12}^{2} f_{2}=-k a_{1} a_{2} x+O(\varepsilon) .
$$

The solution to the linear ordinary differential equation (41) is of the form (see reference [13])

$$
f_{1}(x)=C_{1} \sin (\Delta \arcsin \sqrt{p} x)+C_{2} \cos (\Delta \arcsin \sqrt{p} x),
$$

where $C_{1}$ and $C_{2}$ are arbitrary constants,

$$
\Delta=\omega_{11} / \omega_{12}
$$

and

$$
p=\omega_{11}^{2} / 2 h .
$$

Equation (42) is a non-homogeneous version of equation (41). The complementary solution to equation (42) is of the same form as equation (41),

$$
f_{2, \text { comp }}(x)=C_{3} \sin (\Delta \arcsin \sqrt{p} x)+C_{4} \cos (\Delta \arcsin \sqrt{p} x),
$$

where $C_{3}$ and $C_{4}$ are arbitrary constants. A particular solution to equation (42) is

$$
f_{2, p a r t}(x)=\frac{k a_{1} a_{2}}{\omega_{11}^{2}-\omega_{12}^{2}} x .
$$

Combining equations (46) and (47), the solution to equation (42) becomes

$$
f_{2}(x)=f_{2, \text { comp }}(x)+f_{2, \text { part }}(x) .
$$

In order to solve for the constants $C_{1}, C_{2}, C_{2}$ and $C_{4}$, the following conditions will be employed. Comparing equation (41) with equation (30), the boundary condition, equation (31), becomes

$$
f_{1}^{\prime}=\frac{\omega_{12}^{2} f_{1}}{\omega_{11}^{2} X_{1}} \quad \text { at } X_{1}=\sqrt{\frac{2 h}{\omega_{11}^{2}}}>0
$$

at a rest point $\left(X_{1}, Y_{1}\right)$ for which $X_{1}>0$, where $Y_{1}=\varepsilon f_{1}\left(X_{1}\right)$. Similarly,

$$
f_{2}^{\prime}=\frac{\omega_{12}^{2} f_{2}}{\omega_{11}^{2} X_{2}}-k a_{1} a_{2} \quad \text { at } X_{2}=-\sqrt{\frac{2 h}{\omega_{11}^{2}}}<0
$$

at a rest point $\left(X_{2}, Y_{2}\right)$ with $X_{2}<0$, where $Y_{2}=\varepsilon f_{2}\left(X_{2}\right)$. The modal curve runs from $\left(X_{1}, Y_{1}\right)$ to $\left(X_{2}, Y_{2}\right)$.

Using the normality conditions (equations (49) and (50)) in equations (43-48), we obtain

$$
\begin{gathered}
C_{1} / C_{2}=\tan (\Delta \pi / 2) \equiv Q \quad \text { for } x_{1}>0, \\
C_{3} / C_{4}=-\tan (\Delta \pi / 2)=-Q \quad \text { for } x_{1}<0 .
\end{gathered}
$$


Two more conditions are obtained by requiring that the modal curve be continuous and have a continuous first derivative at $x_{1}=0$. Let $\left(x^{*}, y^{*}\right)$ be the point at which $x_{1}=0$. The matching conditions at the point $x_{1}=0$ gives that

$$
y^{*}=-\frac{a_{1}}{a_{2}} x^{*}=\varepsilon f_{1}\left(x^{*}\right), \quad y^{*}=-\frac{a_{1}}{a_{2}} x^{*}=\varepsilon f_{2}\left(x^{*}\right)
$$

and

$$
f_{1}^{\prime}\left(x^{*}\right)=f_{2}^{\prime}\left(x^{*}\right) .
$$

Solving equation (51) for $C_{1}$, and substituting into equation (53), permits us to obtain the equation for $C_{2}$ in terms of $x^{*}$. Similarly, solving equation (52) for $C_{3}$ and substituting into equation (54) gives an equation for $C_{4}$ in terms of $x^{*}$. When these expressions are substituted into equation (55), we obtain a resulting equation for $x^{*}$ as

$$
\begin{gathered}
x^{*}\left\{\frac{\left[1-k \varepsilon a_{2}^{2} /\left(\omega_{11}^{2}-\omega_{12}^{2}\right)\right](Q \cos z+\sin z)}{-Q \sin z+\cos z}+\frac{Q \cos z-\sin z}{Q \sin z+\cos z}\right\} \\
-\frac{k \varepsilon a_{2}^{2} \sqrt{1-p x^{* 2}}}{\left(\omega_{11}^{2}-\omega_{12}^{2}\right) \Delta \sqrt{p}}=0,
\end{gathered}
$$

where $z=\Delta \sin \sqrt{p} x^{*}$. Equation (56) can be solved using a Taylor series approximation. Since, from equation (56), $x^{*}=0$ when $\varepsilon=0$, we write

$$
x^{*}=\varepsilon x_{1}^{*}+O\left(\varepsilon^{2}\right) .
$$

Substituting equation (57) into equation (56), and using the computer algebra package MACSYMA to Taylor expand in $\varepsilon$, we may collect terms and solve for $x_{1}^{*}$. Once $x^{*}$ is obtained all the constants $\left(C_{1}, C_{2}, C_{3}\right.$ and $\left.C_{4}\right)$ can be calculated. This procedure results in an algebraic approximation for the associated non-linear normal mode. An example calculation will be presented in the next section.

To obtain the period associated with the non-linear normal mode, the equations of motion need to be integrated. For the region $x_{1}>0$, equation (33) can be integrated from $x=X_{1}$ to $x=x^{*}$ to give

$$
t_{1}=\frac{1}{\omega_{11}} \operatorname{arcos}\left(\frac{x^{*}}{X_{1}}\right)
$$

and, similarly, equation (36) can be integrated from $x=X_{2}$ to $x=x^{*}$ to give

$$
t_{2}=\frac{1}{\sqrt{\omega_{11}^{2}+a_{1}^{2} k \varepsilon}} \arccos \left(\frac{x^{*}}{X_{2}}\right) .
$$

Thus the associated period of the motion can be written as

$$
T_{p}=2\left(t_{1}+t_{2}\right) \text {. }
$$

Note that $t_{1}$ and $t_{2}$ depend on $x^{*}$, which is obtained as a Taylor series approximation (57) to equation (56). This period could then be compared with the "bilinear period" defined for the 2-DOF piecewise-linear system.

In order to obtain an approximation for the other non-linear normal mode in this 2-DOF piecewise-linear system, we may interchange the roles of $x$ and $y$ throughout, and utilize a similar perturbation calculation for $x=\varepsilon g(y)$. 


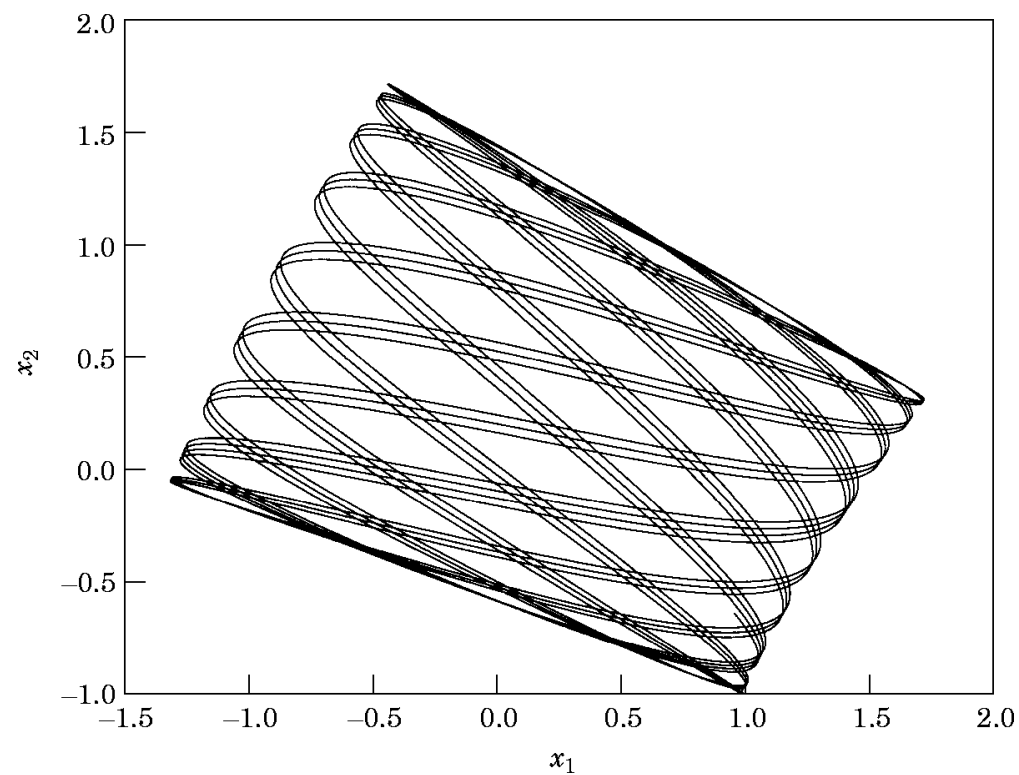

Figure 12. The motion of a phase point in the $x_{1}-x_{2}$ plane for a general motion, not a non-linear normal mode.

\section{NUMERICAL RESULTS}

The results obtained using the perturbation method will be presented for the 2-DOF piecewise-linear system. The values for the parameters in the 2-DOF model (Figure 10) are chosen as $k_{1}=k_{3}=1 \cdot 0$ and $m_{1}=m_{2}=1 \cdot 0$, and the spring constant $k_{2}$ is varied. The equation for the non-linear normal mode is computed to be (cf. equation (40)):

$$
f_{1}(x)=-0 \cdot 0874032 \sqrt{h} k \sin (\Delta \arcsin \sqrt{p} x)-0 \cdot 0597945 \sqrt{h} k \cos (\Delta \arcsin \sqrt{p} x),
$$

$f_{2}(x)=0.0874032 \sqrt{h} k \sin (\Delta \arcsin \sqrt{p} x)-0.0597945 \sqrt{h} k \cos (\Delta \arcsin \sqrt{p} x)-0 \cdot 2 k x$, where $\Delta=2 \cdot 618034$ and $p=0 \cdot 190983 / h$.

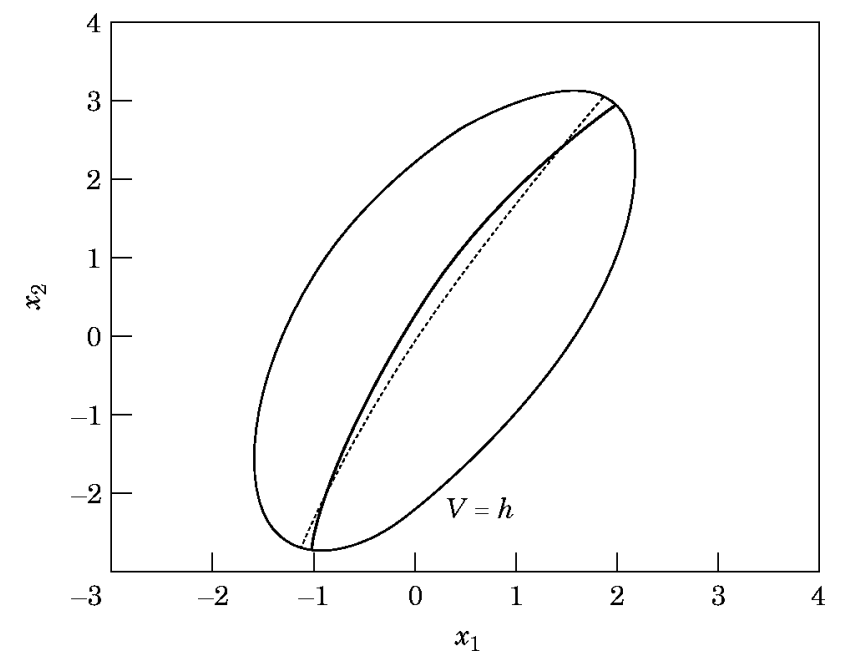

Figure 13. The first mode: $x_{1}$ versus $x_{2}$. The dashed curve represents the eigenvectors in each of the linear regions. Note that the bilinear frequency is a better approximation than the mode shape. - - Non-linear normal mode; $\cdots$, bilinear mode shape. 


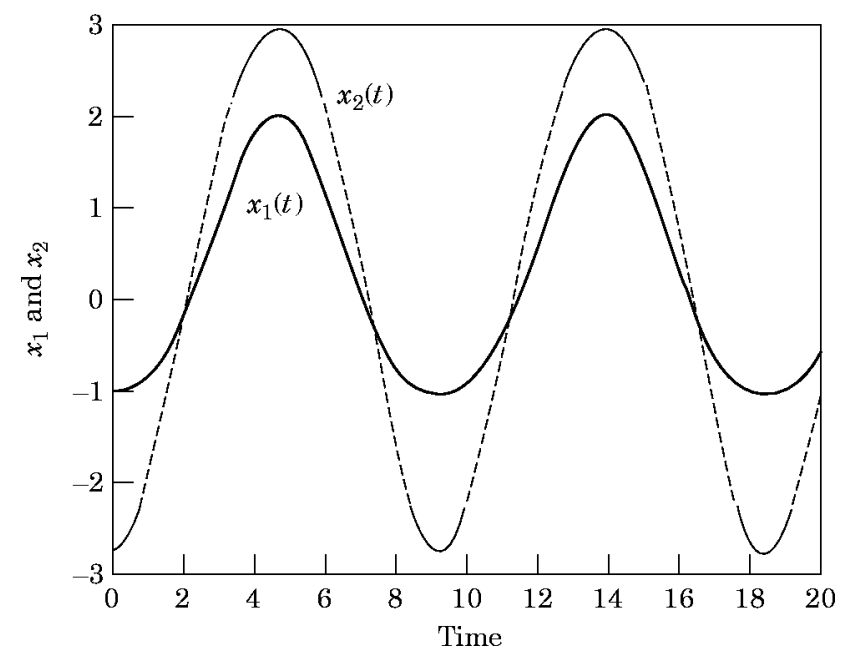

Figure 14. The first mode: —. $x_{1} ;---, x_{2}$; versus time.

In order to verify the results obtained by the perturbation method, the equations of motion are integrated numerically using the fourth order Runge-Kutta method. For any given initial conditions $\left(x_{0}, \dot{x}_{0}, y_{0}, \dot{y}_{0}\right)$, the equations of motion can be integrated numerically. To obtain the modal curve, we need to choose an approximate starting point on the $V=h$ curve. However, the process of choosing the appropriate initial conditions for numerical simulation is that of trial and error. If any other initial condition is chosen the motion of the phase point is quasi-periodic, as shown in Figure 12. In Figures 13 and 15 are shown the non-linear normal modes, obtained using numerical integration, and the bilinear mode shapes for $k_{2}=1 \cdot 0$. In Figures 13 and 15, the eigenvectors of the individual linear modes (i.e., the modes for $x_{1}>0$ and $x_{1}<0$ ) are shown as dotted lines. The computation of the bilinear frequency is based upon approximating actual non-linear

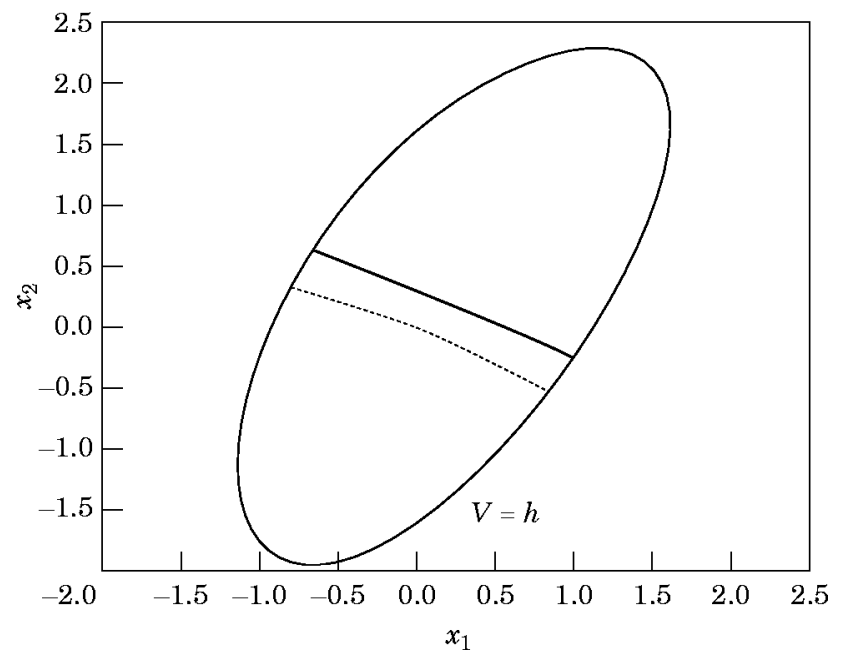

Figure 15. The second mode: $x_{1}$ versus $x_{2}$. The dashed curve represents the eigenvectors in each of the linear regions. Note that the bilinear frequency is a better approximation than the mode shape. - , Non-linear normal mode, $\cdots$, bilinear mode shape. 


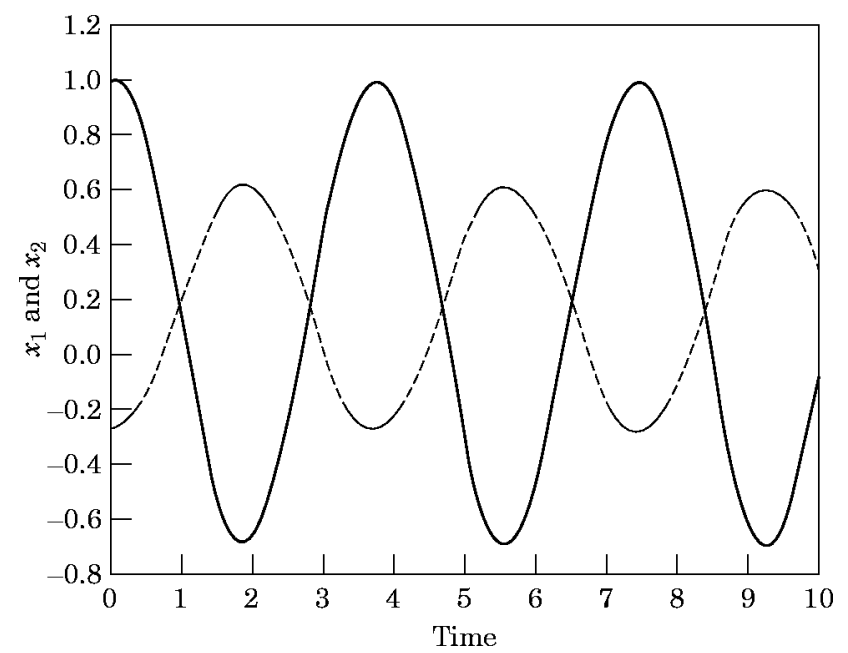

Figure 16. The second mode:,$- x_{1} ;---, x_{2}$; versus time.

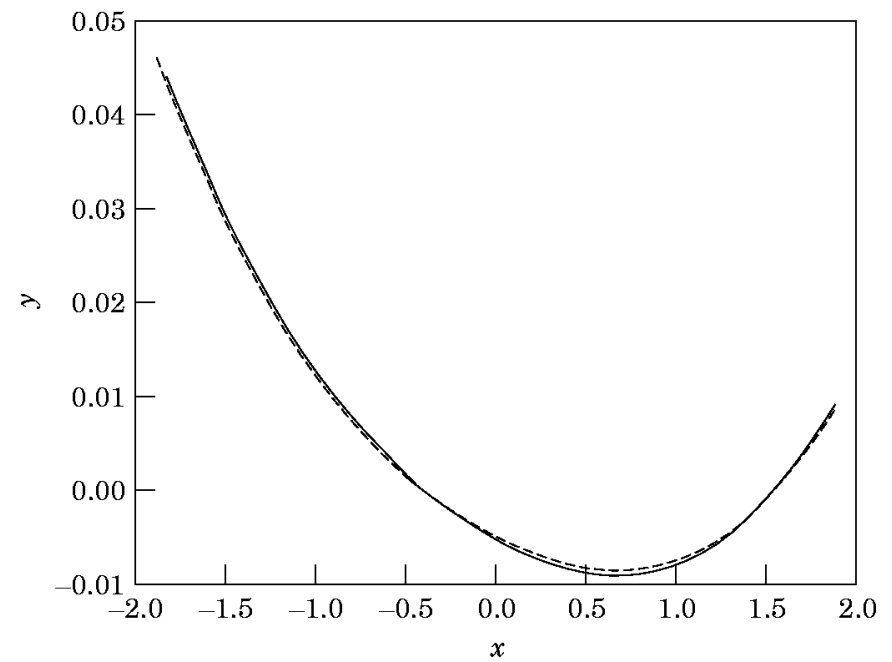

Figure 17. A comparison of the numerical integration and perturbation methods: $k_{2}=0 \cdot 1 .-$, Numerical integration; ---, perturbation method.

normal modes by these bilinear shapes. Note that these non-linear normal modes do not pass through the origin $x_{1}=x_{2}=0$. This could be attributed to the fact, that, due to the piecewise-linearity, the bounding curve $V=h$ and associated vector field is unsymmetric

TABLE 4

A comparison of the period (s) using various methods

\begin{tabular}{cccc}
\hline$k_{2}$ & Bilinear formula & Numerical integration & Perturbation method \\
\hline $0 \cdot 01$ & $10 \cdot 148$ & $10 \cdot 148$ & $10 \cdot 148$ \\
$0 \cdot 1$ & $9 \cdot 997$ & $9 \cdot 997$ & 9.991 \\
$0 \cdot 5$ & $9 \cdot 526$ & $9 \cdot 530$ & $9 \cdot 429$ \\
$1 \cdot 0$ & $9 \cdot 188$ & $9 \cdot 201$ & 8.922 \\
\hline
\end{tabular}




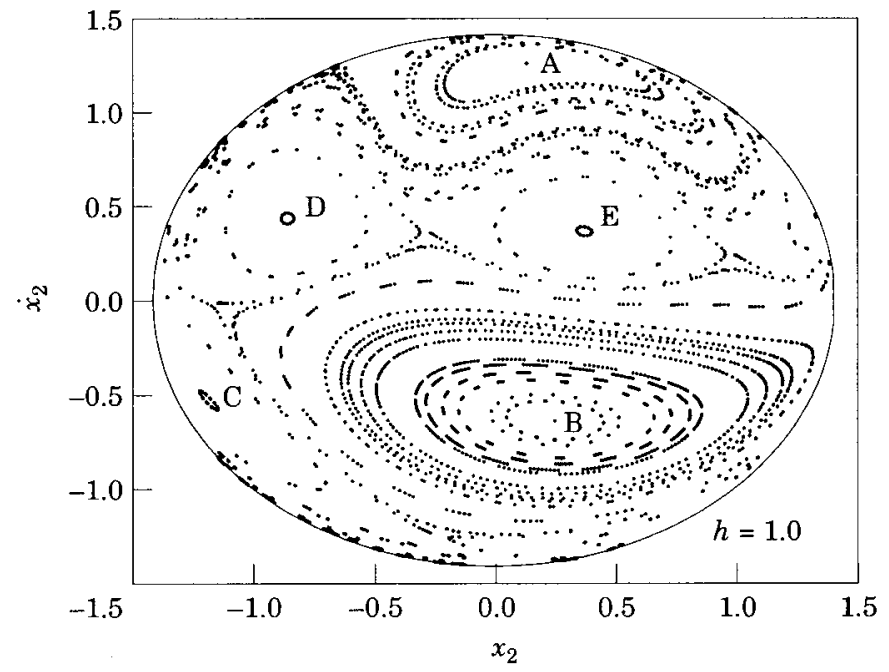

Figure 18. The Poincaré map for $k_{2}=1 \cdot 0, h=1 \cdot 0$ and surface of section $x_{1}=0, \dot{x}_{1}>0 ; h$ is the total energy of the conservative system.

about the origin. The corresponding motions of $x_{1}$ and $x_{2}$ with time are shown in Figures 14 and 16 .

A comparison of the non-linear normal modes obtained by the perturbation method, and by numerical integration, is presented in Figure 17 for $k_{2}=0 \cdot 1$. We see that the resultsagree well. The mismatch between the perturbation method and the numerical results could be attributed to the fact that the results of the perturbation method are based on an order $\varepsilon$ approximation.

In Table 4 we present a comparison of the period associated with the first non-linear normal mode obtained using the bilinear formula, numerical integration and the perturbation method. It is clearly seen that the bilinear formula provides a good approximation for the period associated with the non-linear normal mode. The perturbation method is seen to do well, as expected, for small values of $k_{2}$.

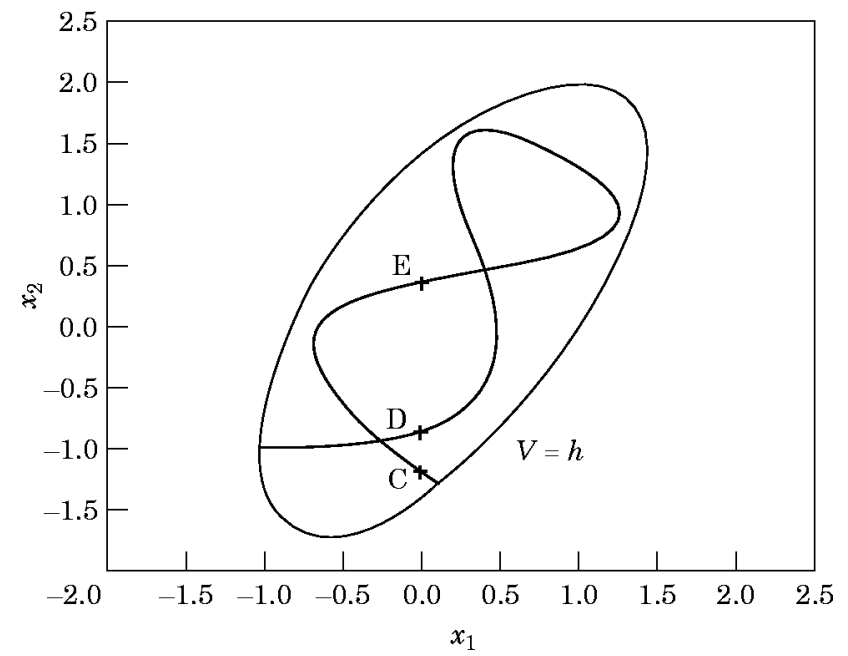

Figure 19. A special kind of non-linear normal mode corresponding to points C, D and E in Figure 18. $V$ is the potential energy of the system and $h$ is the total energy of the system. 


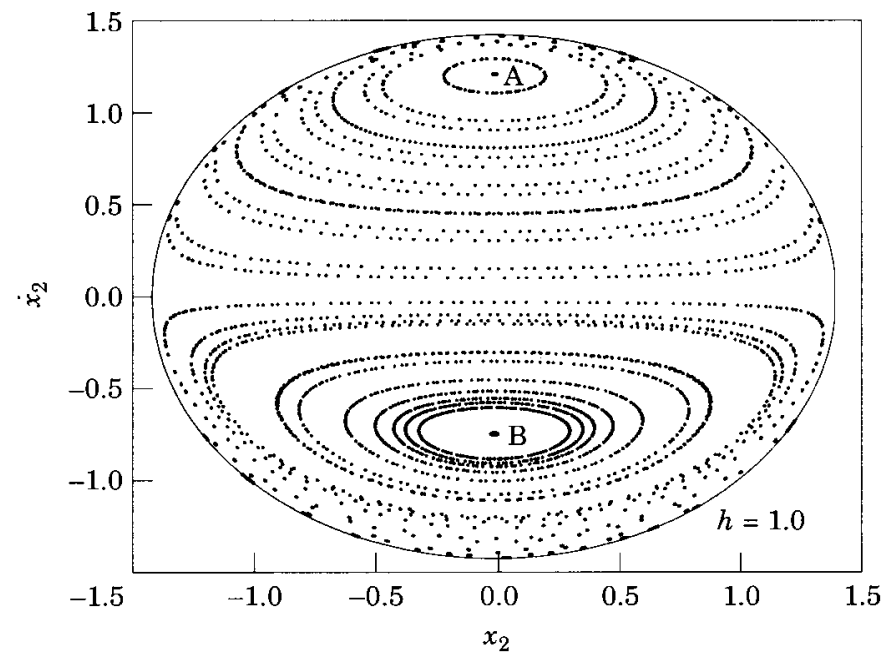

Figure 20. The Poincaré map for $k_{2}=0 \cdot 0, h=1 \cdot 0$ and surface of section $x_{1}=0, \dot{x}_{1}>0$. This figure corresponds to a linear system; in contrast to Figure 18, which corresponds to a piecewise-linear system: $h$ is the total energy of the system.

Our understanding of the non-linear dynamics of this piecewise-linear system may be further enhanced by examining a numerically generated Poincaré map corresponding to total energy $h=1.0$ and a surface of section $x_{1}=0, \dot{x}_{1}>0$ (see reference [18]). The non-linear normal modes that we have investigated in the foregoing correspond to the fixed points A and B in the Poincaré map of Figure 18. We note that, in addition to these priodic motions, we expect to see other periodic motions in this piecewise-linear system. As an example, see Figure 18, where C, D and E lie on a 3-cycle. This motion corresponds to a kind of non-linear normal mode; see Figure 19. However, the associated linear system with $k_{2}=0$ would exhibit no such periodic motion; see Figure 20 .

Generalizing this phenomenon from the 2-DOF system to the infinite-dimensional cracked beam, we expect in the case of the latter that there will exist additional non-linear

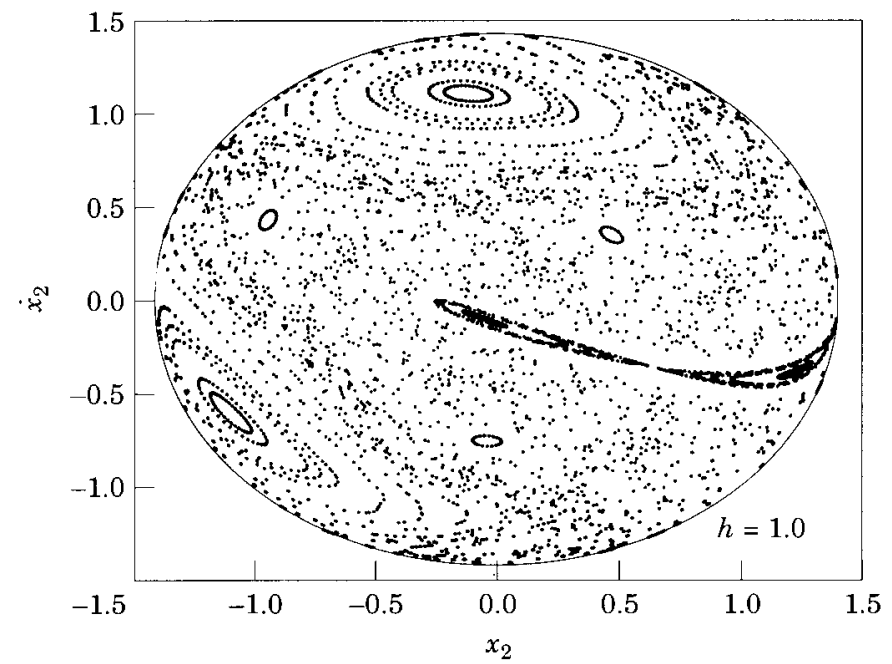

Figure 21. The Poincaré map for $k_{2}=10 \cdot 0, h=1 \cdot 0$ and surface of section $x_{1}=0, \dot{x}_{1}>0: h$ is the total energy of the system. 
normal modes to those discussed in this paper (i.e., in addition to those associated with the bilinear frequencies, $\Omega_{i}$; equation (16)). Their significance is that resonance is expected to occur in their neighborhood, i.e., at forcing frequencies close to their natural frequencies. In addition, we expect that chaotic behavior will occur for large crack depths, in analogy to the behavior of the 2-DOF system for large $k_{2}$; cf., Figure 21.

\section{CONCLUSIONS}

The main goal of this paper is to understand cracked beam dynamics. An eigenvalue problem cannot be formulated for the cracked beam, due to the non-linear boundary conditions associated with the crack being open or closed. However, by modelling the cracked beam as two linear configurations (crack open and crack closed), a definition for the effective natural frequency, called the "bilinear frequency", is developed.

The bilinear period is obtained by adding the half-periods of the individual linear modes in the constrained and unconstrained configurations. The reason that this is an approximation rather than an exact result is as follows: if initial conditions were chosen corresponding to the $i$ th linear normal mode of the unconstrained configuration then, when the crack first closes, this would give rise to a motion in the constrained configuration which would in general consist of a superposition of all associated linear modes rather than just the $i$ th constrained mode. However, we have shown that if the initial conditions were appropriately chosen, a periodic motion would result. If the crack depth is not too large, this initial condition would be close to that of the $i$ th unconstrained mode, and the resulting frequency would be close to the $i$ th bilinear frequency. Furthermore, the use of the bilinear frequency as the effective natural frequency was justified through a systematic study of a simplified model of the cracked beam.

A two-degree-of-freedom piecewise-linear system was studied in order to model and understand the essential non-linear dynamics associated with the cracked beam. The non-linear normal modes associated with the model system and also the associated period are predicted using the perturbation method. It is seen that the bilinear formula is indeed a good approximation for the effective natural frequency, especially when the difference between the linear regions is small, i.e., for small values of $k_{2}$. For large values of $k_{2}$, simulation of the 2-DOF system shows that more complicated motions are possible including chaos.

\section{ACKNOWLEDGMENTS}

This research has been supported by contract number ECS-9321508 of the US National

Science Foundation with Cornell University. The computing for this research was performed at the Cornell National Supercomputer Facility.

\section{REFERENCES}

1. S. Christides and A. D. S. BARr 1984 Journal of Mechanical Sciences 26, 639-648. One-dimensional theory of cracked Bernoulli-Euler beams.

2. M .-H. H. SHEN and C. PIERRE 1990 Journal of Sound and Vibration 138, 115-134. Natural modes of Bernoulli-Euler beams with symmetric cracks.

3. M.-H. H. SHEN and Y. C. CHU 1992 Computers and Structures 45, 79-93. Vibrations of beams with a fatigue crack.

4. Y. C. Chu and M.-H. H. Shen 1992 American Institute of Aeronautics and Astronautics 30, 2512-2519. Analysis of forced bilinear oscillators and the application to cracked beam dynamics. 
5. M. M. F. YUEN 1985 Journal of Sound and Vibration 103, 301-310. A numerical study of the eigenparameter of a damaged cantilever.

6. M.-H. H. ShEn and J. E. TAYlor 1991 Journal of Sound and Vibration 150, 457-484. An identification problem for vibrating cracked beams.

7. W. M. Ostachowicz and M. Krawczuk 1991 Journal of Sound and Vibration 150, 191-201. Analysis of the effect of cracks on the natural frequencies of a cantilever beam.

8. G.-L. Qian S.-N. GU and J.-S. Jiang 1990 Journal of Sound and Vibration 138, 233-243. The dynamic behaviour and crack detection of a beam with a crack.

9. O. N. L. Abraham and J. A. Brandon 1995 Transactions of the American Society of Mechanical Engineers Journal of Vibration, Acoustics, Stress and Reliability in Design 117, 370-377. The modelling of the opening and closing of a crack.

10. K. J. Bathe 1996 Finite Element Procedures. Englewood Cliffs, New Jersey: Prentice-Hall.

11. R. M. Rosenberg 1966 Advances in Applied Mechanics 9, 155-242. On nonlinear vibrations of systems with many degrees of freedom.

12. A. F. Vakakis, L. I. Manevitch, Y. V. Mikhlin, V. N. Pilipchuk and A. A. Zevin 1996 Normal Modes and Localization in Nonlinear Systems. New York: John Wiley.

13. R. H. RAND 1971 International Journal of Nonlinear Mechanics 6, 545-547. A higher order approximation for nonlinear normal modes in two degree of freedom systems.

14. R. H. Rand, C. H. PaK and A. F. Vakakis 1992 Acta Mechanica Supplement 3, 129-145. Bifurcation of nonlinear normal modes in a class of two degree of freedom systems.

15. M. E. KING and A. F. VAKaKis 1994 Journal of Vibration and Acoustics 116, 332-340. An energy-based formulation for computing nonlinear normal modes in undamped continuous systems.

16. A. H. Nayfeh, C. Chin and S. A. Nayfeh 1995 Journal of Vibration of Acoustics 117, 477-481. Nonlinear normal modes of a cantilever beam.

17. S. W. Shaw and C. Pierre 1993 Journal of Sound and Vibration 164, 85-124. Normal modes for nonlinear vibratory systems.

18. A. J. Lichtenberg and M. A. Lieberman 1983 Regular and Stochastic Motion. New York: Springer-Verlag.

19. S. L. Chen and S. W. SHaw 1996 Nonlinear Dynamics 10, 135-164. Normal modes for piecewise linear vibratory systems. 